\title{
Involvement of Fanconi anemia genes FANCD2 and FANCF in the molecular basis of drug resistance in leukemia
}

\author{
CHENJIAO YAO ${ }^{1,2}$, WEI DU ${ }^{2}$, HAIBIUG $\mathrm{CHEN}^{2}$, SHENG XIAO $^{2}$, LIHUA HUANG ${ }^{2}$ and FANG-PING CHEN ${ }^{1,2}$ \\ ${ }^{1}$ Department of Hematology, Xiangya Hospital of Central South University, Changsha, Hunan 410008; \\ ${ }^{2}$ Department of Hematology, Third Xiangya Hospital of Central South University, Changsha, Hunan 410013, P.R. China
}

Received February 17, 2014; Accepted December 9, 2014

DOI: $10.3892 / \mathrm{mmr} .2015 .3288$

\begin{abstract}
The Fanconi anemia (FA)-associated proteins FANCF and FANCD2 are important components of the FA pathway of DNA crosslink repair. FANCF and FANCD2 have been found to be involved in drug-resistant multiple myeloma, ovarian cancer, non-small-cell lung cancer, and head and neck cancer. However, it is unclear whether these two genes participate in adriamycin (ADR)-resistant leukemia. Therefore, the aim of the current study was to investigate FANCF and FANCD2 expression in drug-resistant and drug-sensitive leukemia cells. Western blot analysis revealed enhanced FANCF expression and monoubiquitination of FANCD2 in ADR-resistant cells. Additionally, it was observed that drug-resistant cells had reduced DNA damage compared with drug-sensitive cells. The results of this study indicate that the FA pathway may confer leukemia resistance to ADR via enhanced DNA interstrand crosslink repair.
\end{abstract}

\section{Introduction}

Leukemia is a malignant clonal hematopoietic stem cell disease; leukemia cells are arrested at various cell development stages due to enhanced self-renewal, out of control proliferation, differentiation disorder and blocked apoptosis (1). In the bone marrow and other hematopoietic tissues, leukemia cells proliferate rapidly, inhibiting normal hematopoiesis and infiltrating other tissues and organs. In malignant tumor mortality rankings, leukemia-associated mortality is sixth most common in males, eighth in females, and first in individuals under the age of 35 (2). The primary treatment for leukemia is chemotherapy; however, treatment failure and drug-resistance of cancer cells often occur. Adriamycin (ADR) is a commonly used chemotherapeutic agent used in the treatment of leukemia. ADR inhibits cancer cell

Correspondence to: Professor Fang-Ping Chen, Department of Hematology, Xiangya Hospital of Central South University, No. 87 Xiangya Road, Changsha, Hunan 410008, P.R. China E-mail: jj10888@163.com

Key words: FANCD2, FANCF, leukemia, drug-resistance, Fanconi anemia pathway cycle progression by inducing DNA damage and disordering DNA replication. ADR inserts between the base pairs of DNA in the anthraquinone plane to form specific structures, termed interstrand crosslinks (ICLs) (3). DNA ICLs can block DNA replication. Therefore, the DNA damage repair mechanism may be an important molecular basis of tumor cell drug-resistance to ADR.

The Fanconi anemia (FA) pathway is an important DNA repair pathway, which can effectively repair DNA interstrand crosslinks (4). Fifteen different complementation groups for FA have been defined (5). The FA pathway consists of three major steps: FA core complex formation, FANCD2 ubiquitination and DNA repair in the downstream FA pathway (6). The FA core complex is composed of eight FA proteins. The primary function of the FA core complex is to monoubiquitinate FANCD2, a key step in the FA pathway. Monoubiquitined FANCD2 is able to activate nucleases in the downstream FA pathway and subsequently repair DNA damage, such as ICLs. Chen et al (7) found that overexpression of FANCF in drug-sensitive cells partially reproduced the drug-resistant cell phenotype. The ubiquitination of FANCD2 has an important role in drug resistance in multiple myeloma (8). FANCD2 monoubiquitination and FANCD2 nuclear foci formation are increased in TMZ/BCNU-resistant glioma cells (9). In addition, FANCD2 ubiquitination and FANCF demethylation have been shown to be associated with cisplatinum resistance of ovarian carcinoma cells (10). The aim of the current study was to explore the roles of FANCD2 and FANCF, members of the FA pathway, in ADR-resistant leukemia cells.

\section{Materials and methods}

Cell lines and drugs. The K562 and K562/R human leukemia cell lines were obtained from the Cell Center of Xiangya School of Medicine (Changsha, China). Cell lines were grown in RPMI-1640 medium (Hyclone, Logan, UT, USA) supplemented with $10 \%$ heat-inactivated fetal bovine serum (Evergreen, Suzhou, China). K562/R ADR-resistant cells were grown in culture medium containing $1 \mu \mathrm{M}$ ADR (Zhejiang Haizheng Pharmaceutical, Taizhou, China) to maintain drug-resistance.

Methyl-thiazoltetrazolium (MTT) cytotoxicity assay. Cells were seeded at a density of 5,000 cells/well in 96-well plates (Costar, 
Corning, NY, USA). To establish a dose response to ADR, cells were incubated with ADR in 2-fold serial dilutions ranging from 1 to $64 \mu \mathrm{mol} / 1$. The blank group did not contain cells. Each group included three parallel samples. The total medium volume of each well was $200 \mu$ l. Two days after drug addition, $20 \mu \mathrm{l}$ MTT solution $[5.0 \mathrm{~g} / 1$ in phosphate-buffered saline (PBS; ZSGB-BIO, Beijing, China)] was added. After $4 \mathrm{~h}, 100 \mu \mathrm{l}$ dimethylsulfoxide (Sigma-Aldrich, St. Louis, MO, USA) was added per well and the cells were allowed to stand for $30 \mathrm{~min}$ prior to detection.

Cell cycle analysis. Propidium iodide (PI; Sigma-Aldrich) staining was used to measure the effects of ADR on cell-cycle progression. K562 and K562/R cells were treated with 2 or $20 \mu \mathrm{mol} / \mathrm{l} \mathrm{ADR}$ for $2 \mathrm{~h}$ and cultured in drug-free medium; cells were collected at 24 and $48 \mathrm{~h}$. Subsequently, cells were fixed with $70 \%$ ethanol at $4^{\circ} \mathrm{C}$ overnight, resuspended in $500 \mu \mathrm{lPBS}$ containing $500 \mu \mathrm{g} / \mathrm{ml} \mathrm{PI}$ and $10 \mathrm{mg} / \mathrm{ml}$ RNase A (Invitrogen Life Technologies, Carlsbad, CA, USA), and incubated at $37^{\circ} \mathrm{C}$ for $30 \mathrm{~min}$ in the dark. The cell cycle was analyzed by flow cytometry (MofloXDP; Beckman Coulter, Brea, CA, USA) using FlowJo 4.4.4 software (Tree Star, Inc., Ashland, OR, USA).

Reverse transcription-quantitative polymerase chain reaction (RT-qPCR). TotalRNA Extractor (Shenggong), ReverTra Ace qPCR RT-kit (Toyobo Co., Ltd., Osaka, Japan) and SYBR Green ${ }^{\circledR}$ Realtime PCR Master mix (Toyobo Co., Ltd.) were used for total RNA $(1 \mu \mathrm{g})$ extraction, cDNA synthesis and PCR analysis, respectively. qPCR of FANCF (forward, 5'-CTCCTGTCTATCTGGGTCTGCT-3' and reverse, 5'-GAGGCTTTGAAACCTATTGTGC-3') and FANCD2 (forward, 5'-AGCAATGTATGCCGCTCCT3' and reverse, 5'-TTGGGTGAGTCTCGTGTCC-3') was run for 40 cycles. Each cycle consisted of denaturation $\left(30 \mathrm{sec}\right.$ at $95^{\circ} \mathrm{C}$; first cycle, $2 \mathrm{~min} 30 \mathrm{sec}$ ), annealing $\left(30 \mathrm{sec}\right.$ at $\left.58^{\circ} \mathrm{C}\right)$ and extension (30 sec at $72^{\circ} \mathrm{C}$; last cycle $7 \mathrm{~min} 30 \mathrm{sec}$ ). The gene expression level was normalized using the endogenous control gene GAPDH. qPCR reactions were performed using Mastercycler ep realplex (Eppendorf, Hamburg, Germany).

Western blot analysis. FANCD2 and FANCF proteins were detected by western blot analysis. Cells were lysed using a commercial lysis buffer kit (Beyotime Institute of Biotechnology, Haimen, China) according to the manufacturer's instructions. Following quantification of proteins using a BCA kit (Beyotime, Shanghai, China), equal quantities of protein lysates were mixed with SDS-PAGE protein loading buffer, boiled for $5 \mathrm{~min}$, and then run on $10 \%$ SDS-PAGE at $100 \mathrm{~V}$ for $180 \mathrm{~min}$ and transferred onto a polyvinylidine fluoride membrane (Beyotime Insitute of Biotechnology). The primary antibodies used for western blotting were mouse monoclonal immunoglobulin $\mathrm{G}_{1}\left(\mathrm{IgG}_{1}\right)$ anti-human FANCF (sc-271952; Santa Cruz Biotechnology, Dallas, TX, USA), rabbit polyclonal IgG anti-human FANCD2 (sc-28194; Santa Cruz Biotechnology) and $\beta$-actin (Sigma-Aldrich). The secondary antibodies were polyclonal horseradish peroxidase-conjugated AffiniPure goat anti-rabbit IgG, Fc fragment specific (111-035-008), polyclonal horseradish peroxidase-conjugated AffiniPure goat anti-rabbit $\operatorname{IgG}(\mathrm{H}+\mathrm{L})$ (111-035-003) and polyclonal rhodamine (TRITC) AffiniPure
Goat Anti-Mouse IgG (H+L) (115-025-062) (Jackson ImmunoResearch Laboratories, Inc., West Grove, PA, USA). Results were visualized using a DAB Horseradish Peroxidase Color Development kit (Beyotime Institute of Biotechnology) for chemiluminescent detection.

Alkaline comet assay. The alkaline comet assay was used to detect ADR-induced DNA cross-links in drug-sensitive (K562) and drug-resistant (K562/R) leukemia cells. A total of $1 \times 10^{5}$ cells were treated with $80 \mu \mathrm{M}$ ADR or vehicle control for $24 \mathrm{~h}$, washed in PBS and resuspended in ice-cold PBS. A volume of $\sim 10 \mu \mathrm{l}$ of the resuspended cells was mixed with $65 \mu \mathrm{l}$ of low melting point agarose at $4^{\circ} \mathrm{C}$. The slides were placed in the dark at $4^{\circ} \mathrm{C}$ until gelling occurred and then immersed in prechilled lysis buffer (Jingcai, Xi'an, China) at $4^{\circ} \mathrm{C}$. After a 60-min incubation, the buffer was aspirated and replaced with pre-chilled alkaline solution for 90-120 min at $4^{\circ} \mathrm{C}$. After lysis and unwinding, the slides were placed in a horizontal electrophoresis tank filled with freshly prepared alkaline electrophoresis buffer (Jingcai). The electrophoresis was run for $30 \mathrm{~min}$ at $25 \mathrm{~V}$ and $300 \mathrm{~mA}$. After electrophoresis, the slides were transferred to neutralization buffer (Jingcai) for $10 \mathrm{~min}$. The neutralization buffer was aspirated and replaced three times. Thereafter, the slides were allowed to air dry and $50 \mu \mathrm{l} /$ well PI $(5 \mu \mathrm{g} / \mathrm{ml})$ was added to each slide for $5 \mathrm{~min}$ in the dark at room temperature, for DNA staining. DNA migration was observed using a fluorescence microscope (AE31; Motic, Hong Kong, China). For each sample, 200 randomly selected cells were analyzed using CASPLab version 1.2.2 (Comet Assay Software Project, Wrocław, Poland).

Statistical analysis. Statistical analysis was performed using Student's t-test or one-way analysis of variance with SPSS version 21.0 (IBM, Armonk, NY, USA). P<0.05 was considered to indicate a statistically significant difference.

\section{Results}

Drug-resistant cells have a higher survival rate after ADR treatment. An MTT assay was used to compare ADR sensitivity in the drug-sensitive (K562) and drug-resistant (K562/R) leukemia cell lines. The $\mathrm{IC}_{50}$ of $\mathrm{K} 562 / \mathrm{R}$ cells was $20.6 \pm 4.30 \mu \mathrm{M}$ and the $\mathrm{IC}_{50}$ of $\mathrm{K} 562$ cells was $0.78 \pm 0.05 \mu \mathrm{M}$ (Fig. 1A). ADR-resistant K562/R cells exhibited increased survival (Fig. 1B) compared with that of their drug-sensitive parental K562 cells.

Effect of ADR on cell-cycle progression in K562 and K562/R cells. To measure dose-response and time-response effects of ADR on cell-cycle progression, K562 and K562/R cells were treated with 2 or $20 \mu \mathrm{M}$ ADR for $2 \mathrm{~h}$, cultured in drug-free medium, and collected at 24 and $48 \mathrm{~h}$. After $24 \mathrm{~h}$, high-dose $(20 \mu \mathrm{M})$ and low-dose $(2 \mu \mathrm{M})$ ADR treatment resulted in an accumulation of $40.7 \pm 0.55$ and $50.2 \pm 1.35 \%$ K562 cells in the $\mathrm{S}$ phase, respectively (Figs. 2 and 3). K562/R cells were arrested in the G1 phase $(94.7 \pm 1.36 \%)$ after high-dose ADR treatment (20 $\mu \mathrm{M})$ (Figs. 2 and 3). However, low-dose ADR treatment $(2 \mu \mathrm{M})$ induced $\mathrm{K} 562 / \mathrm{R}$ cell arrest in the $\mathrm{S}$ phase $(59.19 \pm 2.71 \%)$. Following ADR $(20 \mu \mathrm{M})$ treatment for 24 or 48 h, K562 cells were arrested in the S phase $50.2 \pm 1.35 \%$ and 
A

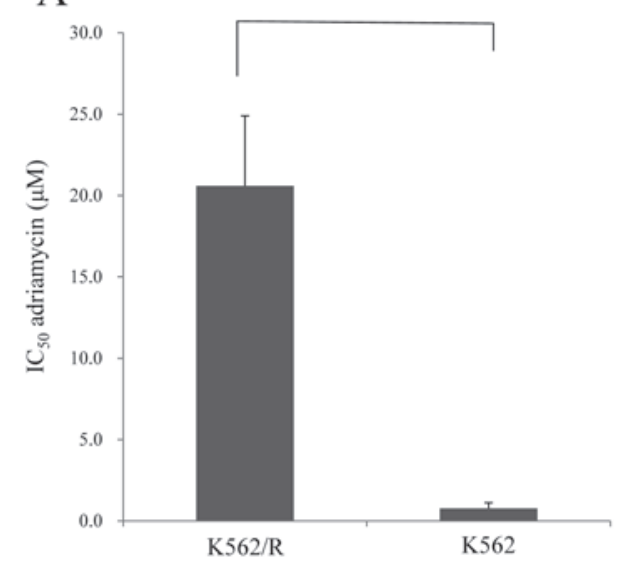

B

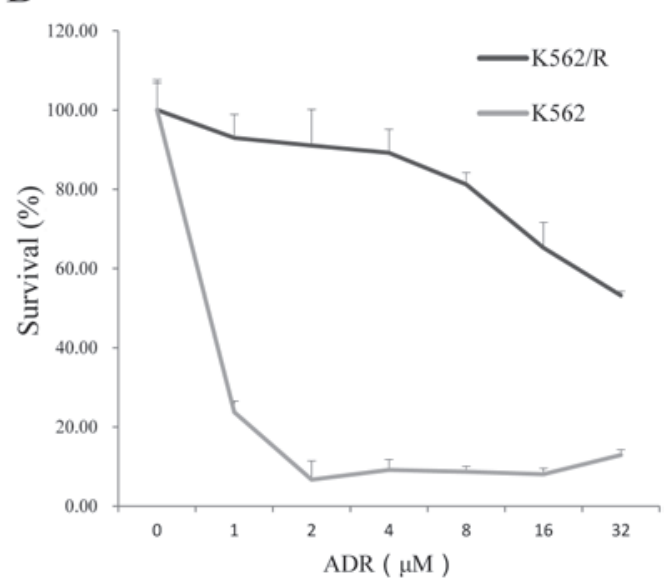

Figure 1. Methyl-thiazoltetrazolium assay. (A) Median inhibitory concentration $\left(\mathrm{IC}_{50}\right)$ values are the mean of three independent experiments \pm standard deviation. (B) Drug-resistant cells exhibited a higher survival rate than drug-sensitive cells. " $\mathrm{P}<0.05$ vs. the K562 cells.

A

$0 \mu \mathrm{M}$

$\mathrm{K} 562 / \mathrm{R}$

$0 \mathrm{~h}$
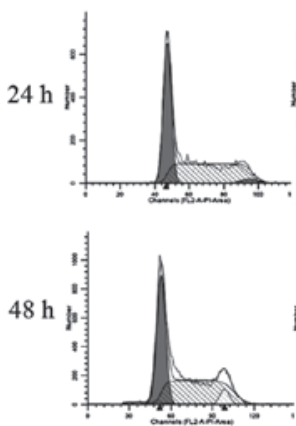

$20 \mu \mathrm{M}$
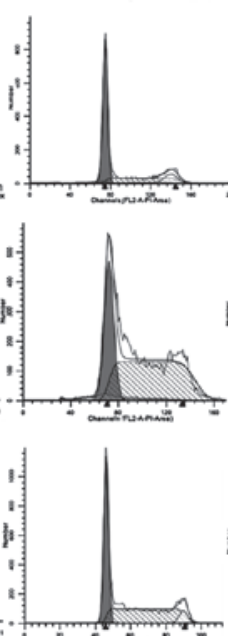

$40 \mu \mathrm{M}$
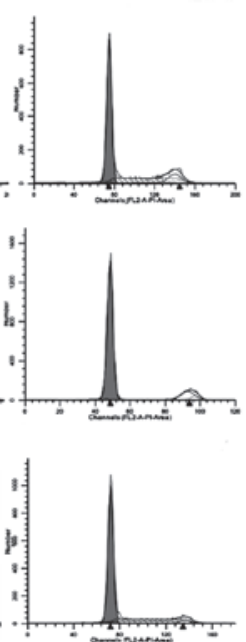

B

$\begin{array}{rr}\mathrm{K} 562 \\ 0 \mu \mathrm{M} & 20 \mu \mathrm{M}\end{array}$
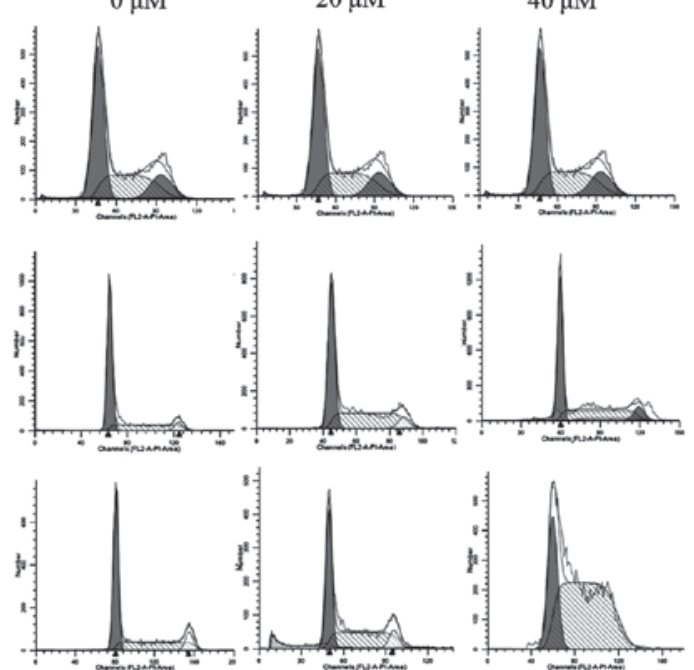

Figure 2. Flow cytometric analysis. Cell cycle distribution of K562 and K562/R cells after adriamycin treatment in (A) K562/R and (B) K562 cells.

A

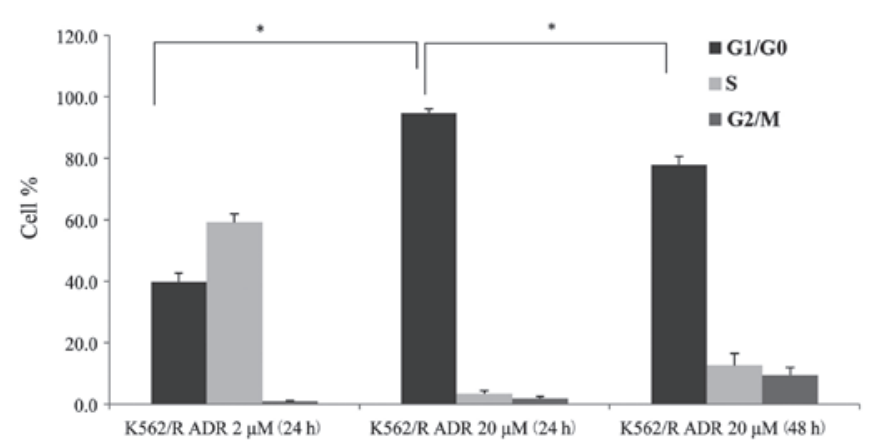

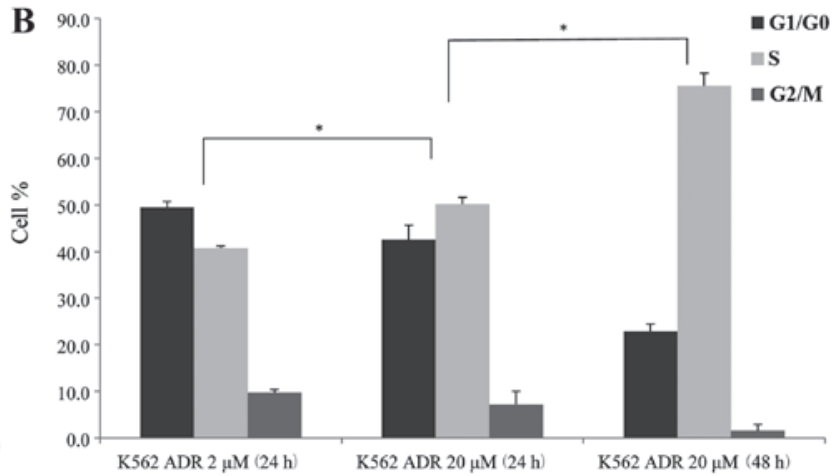

Figure 3. Flow cytometric analysis. The data from flow cytometric analysis shows the dose-response and the time-response effects of ADR on cell-cycle progression of (A) K562/R cells and (B) K562 cells. ${ }^{*} \mathrm{P}<0.05$. ADR, adriamycin.

$75.6 \pm 7.72 \%$, respectively (Fig. 2 and 3). ADR treatment over a longer period resulted in a greater number of K562 cells arresting in the $\mathrm{S}$ phase. K562/R cells were arrested in the G1 phase after ADR $(20 \mu \mathrm{M})$ treatment for $24(94.7 \pm 1.36 \%)$ or $48 \mathrm{~h}(77.9 \pm 2.69 \%)$; however, K562/R cells that were treated for $48 \mathrm{~h}$ had fewer cells in the $\mathrm{G}_{1}$ phase (Figs. 2 and 3). As 

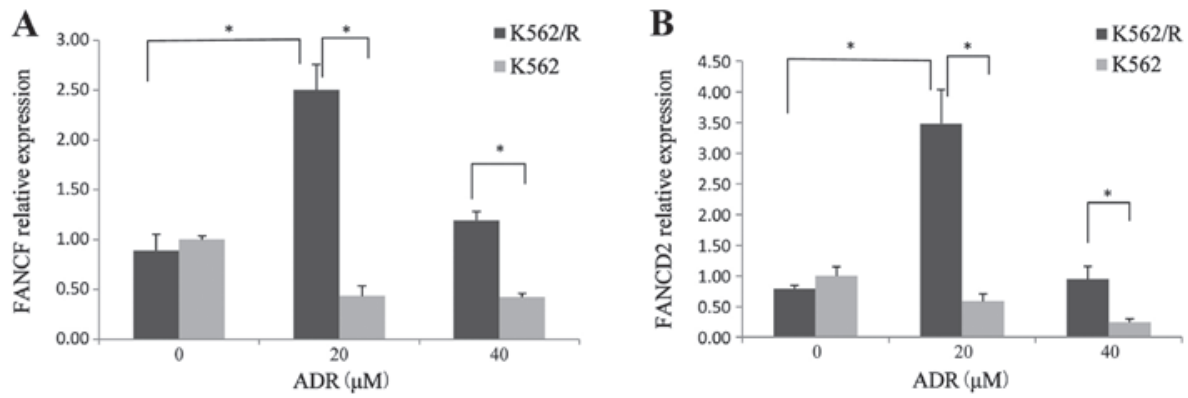

Figure 4. Reverse transcription-quantitative polymerase chain reaction analysis. K562 and K562/R cells were treated with 20 and $40 \mu \mathrm{M}$ ADR and harvested after $24 \mathrm{~h}$. (A) FANCF and (B) FANCD2 gene expression was observed and was shown to be enhanced in drug-resistant cells. " $\mathrm{P}<0.05$. ADR, adriamycin.

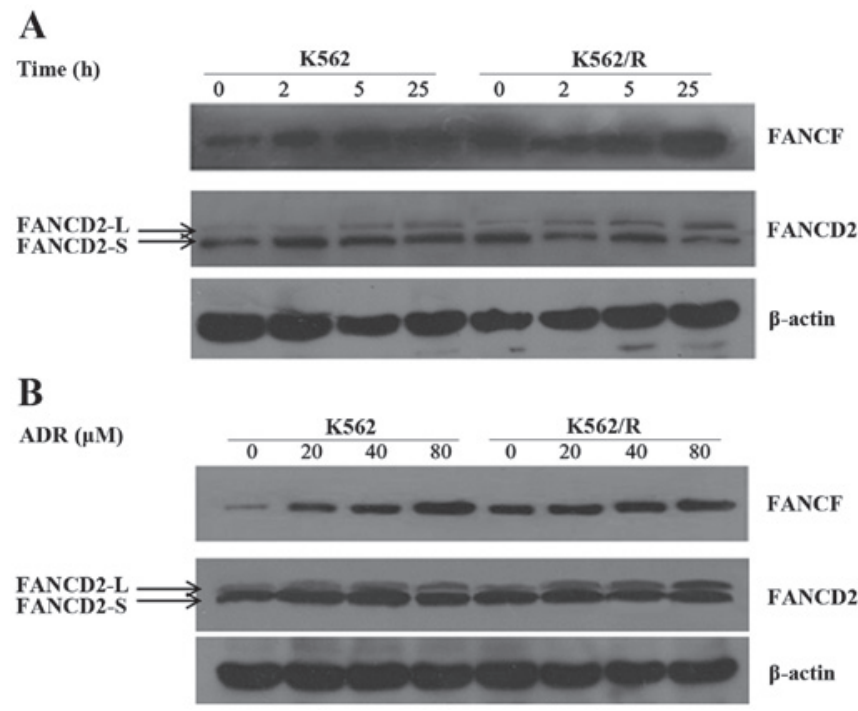

Figure 5. Western blot analysis. FANCD2-L/S and FANCF increased in K562 and K562/R cells following ADR treatment at (A) different time-points or (B) various concentrations. ADR, adriamycin.

time passed, the $\mathrm{G}_{1}$ phase-arresting effect of ADR on K562/R cells became weaker, however, the effect on cell cycle arrest of K562 cells became stronger.

ADR-resistant leukemia cells exhibit enhanced expression of FANCD 2 and FANCF. qPCR showed that K562/R cells had higher levels of FANCF and FANCD2 expression after ADR $(20 \mu \mathrm{M})$ treatment for $24 \mathrm{~h}$ compared with the untreated K562/R cells. ADR (20 and $40 \mu \mathrm{M})$ treatment resulted in greater FANCF and FANCD2 expression in K562/R cells than K562 cells (Fig. 4). The results revealed that the expression of the two components of the FA pathway were upregulated in drug-resistant $\mathrm{K} 562 / \mathrm{R}$ cells compared with drug-sensitive K562 cells.

FANCD2 ubiquitination and FANCF protein expression are enhanced in ADR-resistant leukemia cells. FANCD2 protein has two isomers, namely FANCD2-S and FANCD2-L. FANCD2-S is non-ubiquitinated FANCD2 protein ndFANCD2-L is ubiquitinated FANCD2 protein. In the FA pathway, ubiquitinated FANCD2 proteins are functional; thus, primarily the FANCD2 ubiquitination level was compared with the FANCD2-L/S (the ratio of ubiquitinated FANCD2 protein to non-ubiquitinated FANCD2 protein). FANCD2 is one of the most important proteins in the FA pathway, and FANCF is important for forming the FA core complex. Therefore, FANCD2-L/S and FANCF were monitored at various time points after multi-concentration drug treatment in the two cell lines. The results revealed that the levels of FANCD2-L/S and FANCF were significantly increased within $2 \mathrm{~h}$ of ADR treatment, and the maximum increase of FANCD2-L/S and FANCF occurred within $25 \mathrm{~h}$ after ADR treatment (Fig. 5). Additionally, the results revealed that the level of FANCD2-L/S and FANCF was clearly increased after $20 \mu \mathrm{M}$ ADR treatment for $25 \mathrm{~h}$ and the maximum increase of FANCD2-L/S and FANCF occurred after $80 \mu \mathrm{M}$ ADR treatment for $25 \mathrm{~h}$ (Fig. 5). The level of FANCD2-L/S and FANCF in K562 and K562/R cell lines increased in a timedependent manner and dose-dependent manner, but higher expression levels of FANCD2-L/S and FANCF were observed at all times and doses in drug-resistant cells compared with drug-sensitive cells. In addition, the results suggest that increased FANCD2-L may be due to the enhanced expression of FANCF protein. A stable FA complex functions as the ubiquitin ligase for FANCD2. When the expression of FANCF was increased, the expression of FANCF and monoubiquitinated FANCD2 protein were significantly increased in ADR-resistant leukemia cells. Therefore it was concluded that 


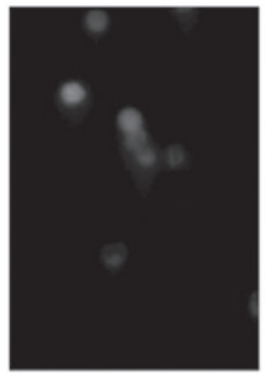

K562

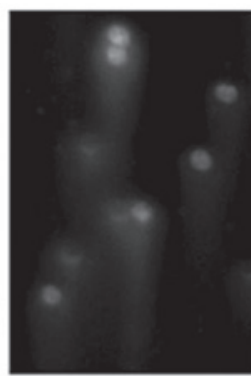

K562+ADR

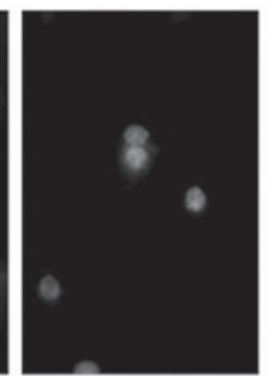

K562/R

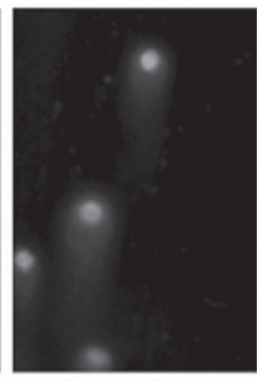

K562/R+ADR

Figure 6. Comet assay. ADR-resistant leukemia cells exhibit less DNA damage than ADR-sensitive cells. Propidium iodide staining (magnification, x100). ADR, adriamycin.

FANCF and ubiquitinated FANCD2 proteins were involved in ADR-resistant leukemia cells.

ADR-resistant cells experience less DNA damage compared with drug-sensitive cells. Photomicrographs of the ADR-induced DNA damage are shown in Fig. 6. The ADR-induced DNA ICL formation effect was obvious in K562 and K562/R cells after ADR treatment. The ICLs were significantly increased in K562 and K562/R cells after ADR treatment compared with those of the group without ADR treatment. However, drug-resistant cells had fewer ICLs than drug-sensitive cells, indicating that ADR-resistant cells exhibited less DNA damage compared with drug-sensitive cells.

\section{Discussion}

Currently the curative effect of ADR for leukemia treatment remains limited. Resistance to ADR is often observed in cancer cells. DNA repair pathway activation is one of the mechanisms for resistance to ADR in tumor cells. A number of chemotherapeutic agents cause DNA damage, and then inhibit tumor cell replication and induce cell apoptosis to achieve their therapeutic effect. The FA pathway is a DNA repair pathway, which repairs DNA in one of three ways: Base excision repair, homologous recombination repair or translesion DNA synthesis. It has been shown that the FA pathway is involved in resistance to a variety of drugs in tumor cells. A total of $15 \mathrm{FA}$ proteins have already been identified, including FANCA, FANCB, FANCC, FANCD1, FANCD2, FANCE, FANCF, FANCG, FANCI, FANCJ, FANCL, FANCM, FANCN, FANCP and RAD51C. The formation of the FA core complex and FANCD2 monubiquitylation are two key steps in the FA pathway. The FA core complex has E3 ligase activity and is composed of eight FA proteins (A, B, C, E, F, G, L and M), FAAP100 protein and FAAP24 protein (11). FANCF protein is an important adapter, which connects the FANCA/FANCG and FANCC/FANCE subunits in the FA core complex (12). It interacts directly with FANCG protein and aids in stabilizing a complex involving FANCA and FANCC proteins using its $\mathrm{C}$-terminal. In addition, FANCF interacts with the FANCC-FANCE sub-complex through the N-terminal region (13). Therefore, FANCF can stabilise the whole FA core complex. When DNA is damaged, the stable FA core complex uses its E3 ubiquitin ligase activity to assist in the ubiquitination of FANCD2. After FANCD2 ubiquitination, the downstream DNA repair enzymes can be recruited to repair DNA damage. Therefore FANCF and FANCD2 were selected to investigate the association between the FA pathway and drug-resistant leukemia.

ADR is an anthracycline cytotoxic drug, it is a non-specific cell cycle drug; however, S-phase tumor cells have a higher sensitivity to it. Its molecular structure can be embedded into the DNA double strand to form a stable complex, leading to the inhibition of DNA replication and RNA synthesis, thereby causing cell death or arrest in the $S$ or $G_{2}$ phase (14). The results of the current study determined that the growth of K562 and K562/R cells was inhibited after ADR treatment. K562 cells were arrested in the $S$ phase and K562/R cells were arrested in $\mathrm{G}_{1}$ phase. The effect of cell cycle arrest was weaker in K562/R cells than in K562 cells. In the process of cell division, the $\mathrm{S}$ phase is primarily responsible for DNA replication; cells were arrested in the $S$ phase, showing that DNA replication was inhibited and required repairing, and the more cells that were blocked in the $\mathrm{S}$ phase, the more DNA damage that required repairing. In the comet assay, drug-sensitive cells had a greater number of DNA ICLs than drug-resistant cells.

The results of the RT-qPCR showed that K562/R drug-resistant cells expressed a higher level of FANCD2 and FANCF mRNA than K562 cells. The experimental results are similar to the results of Yarde et al (15), which revealed that the FA/BRCA pathway is involved in the drug-resistance of multiple myeloma cells. In addition, the current study determined that the protein expression levels of FANCD2-L/S and FANCF in K562 and $\mathrm{K} 562 / \mathrm{R}$ cell lines were increased in a time- and dose dependent manner; however, a higher expression level of FANCD2-L/S and FANCF was observed in drug-resistant cells compared with drug-sensitive cells. Lundholm et al (16) determined that FANCD2 ubiquitination was enhanced in cisplatin-resistant non-small cell lung cancer stem cells. Additionally, the level of FANCD2 ubiquitination has been shown to be reduced in drug-resistant multiple myeloma cells after the reversal of cell drug-resistance (8). Therefore, FANCF and ubiquitinated FANCD2 proteins may be involved in the development of drug-resistance in leukemia cells.

The primary function of the FA pathway is to repair DNA ICLs, therefore, in the current study the DNA damage of the two cell lines was detected using a comet assay after ADR 
treatment. Drug-resistant cells developed fewer DNA ICLs than drug-sensitive cells. Therefore it was hypothesized that drug-resistant cells may have stronger DNA repair ability.

In conclusion, the results of the current study showed that drug-resistant leukemia cells have enhanced FANCF expression and FANCD2 ubiquitination. Therefore, the FA pathway, which FANCF and FANCD2 are involved in, may be a potential mechanism for ADR resistance in leukemia cells.

\section{Acknowledgements}

This study was supported by grants from the Research Foundation of Hunan Provincial Science and Technology Department (no. 2012FJ3138) and the Postgraduates Innovation Foundation of Hunan Province (no. CX2011B065).

\section{References}

1. Wu DP: Leukemia. Internal Medicine 9: 600-616,2009 (In Chinese)

2. Chu JX, Yang CL and Yang TY: Leukemia epidemiology report of China. Tianjing Med J 6: 323-326, 1982 (In Chinese).

3. Wang Y, Xu LH, Duan ZH, Cao KY, Luo JG, Xu Y and Shi PJ: Effects of ADM on cell apoptosis and expression of FAK mRNA in K562. J Trop Med 12: 1195-1198, 2012 (In Chinese).

4. Moldovan GL and D'Andrea AD: How the fanconi anemia pathway guards the genome. Annu Rev Genet 43: 223-249, 2009.

5. Kim H and D'Andrea AD: Regulation of DNA cross-link repair by the Fanconi anemia/BRCA pathway. Genes Dev 26: 1393-1408, 2012.
6. Romick-Rosendale LE, Lui VW, Grandis JR and Wells SI: The Fanconi anemia pathway: repairing the link between DNA damage and squamous cell carcinoma. Mutat Res 743-744: 78-88, 2013.

7. Chen Q, Van der Sluis PC, Boulware D, Hazlehurst LA and Dalton WS: The FA/BRCA pathway is involved in melphalan-induced DNA interstrand cross-link repair and accounts for melphalan resistance in multiple myeloma cells. Blood 106: 698-705, 2005.

8. Xiao H, Xiao Q, Zhang K, Zuo X and Shrestha UK: Reversal of multidrug resistance by curcumin through FA/BRCA pathway in multiple myeloma cell line MOLP-2/R. Ann Hematol 89: 399-404, 2010.

9. Chen CC, Taniguchi T and D'Andrea A: The Fanconi anemia (FA) pathway confers glioma resistance to DNA alkylating agents. J Mol Med (Berl) 85: 497-509, 2007.

10. Taniguchi T, Tischkowitz M, Ameziane N, et al: Disruption of the Fanconi anemia-BRCA pathway in cisplatin-sensitive ovarian tumors. Nat Med 9: 568-574, 2003.

11. D'Andrea AD: Targeting DNA repair pathways in AML. Best Pract Res Clin Haematol 23: 469-473, 2010.

12. Leveille F, Blom E, Medhurst AL, et al: The Fanconi anemia gene product FANCF is a flexible adaptor protein. J Biol Chem 279: 39421-39430, 2004.

13. de Winter JP, van der Weel L, de Groot J, et al: The Fanconi anemia protein FANCF forms a nuclear complex with FANCA, FANCC and FANCG. Hum Mol Genet 9: 2665-2674, 2000.

14. Wang $X$ and Li DH: Adriamycin-induced apoptosis of tumor cells research progress. China Practical Med 07: 247-249, 2010.

15. Yarde DN, Oliveira V, Mathews L, et al: Targeting the Fanconi anemia/BRCA pathway circumvents drug resistance in multiple myeloma. Cancer Res 69: 9367-9375, 2009.

16. Lundholm L, Haag P, Zong D, et al: Resistance to DNA-damaging treatment in non-small cell lung cancer tumor-initiating cells involves reduced DNA-PK/ATM activation and diminished cell cycle arrest. Cell Death Dis 4: e478, 2013. 